\title{
Roma, teatro de las naciones: el artista y la ciudad (1750-1780)
}

\author{
RaQuel Gallego*
}

ROMA, TEATRO DE LAS NACIONES: EL ARTISTA Y LA CIUDAD (1750-1780)

\section{Resumen}

Un riguroso y dilatado estudio de los stati delle anime conservados en el Archivio Storico del Vicariato de Roma nos permite saber en qué parte de la ciudad se establecieron los artistas españoles, los franceses, así como aquellos que procedían del norte de Europa entre 1750 y 1780, una elección que, cómo tendremos ocasión de ver, debió de estar condicionada en muchos casos por la pertenencia a una nación u otra. Además, podremos saber cuándo esta elección experimentó modificaciones y cuáles fueron las razones de las mismas.

\section{Rome, Theatre of Nations: the Artist And the City (1750-1780)}

\begin{abstract}
The in-depth study of the stati delle anime preserved in the Historical Archive of the Vicariate in Rome has proved to be of great importance to our understanding of in which parts of the city Spanish, French and northern European artists typically settled between 1750 and 1780 . Their choices, as we will demonstrate, could well have been influenced by their nationality. Furthermore, through this analysis we can see when these choices changed and understand the reasons behind these changes.
\end{abstract}

GALlego, R., «Roma, teatro de las naciones: el artista y la ciudad (1750-1780)», Acta/Artis. Estudis d'Art Modern, 4-5, 2016-2017, págs. 41-53

Palabras Clave: stati delle anime, Archivio Storico del Vicariato de Roma, nacionalismo, Roma, Grand Tour

KEYwORDs: stati delle anime, Archivio Storico del Vicariato of Rome, nationalism, Rome, Grand Tour

\footnotetext{
* Este trabajo se enmarca en el proyecto de investigación ACAF/ART IV «Cartografías analíticas, críticas y selectivas del entorno artístico y monumental del área mediterránea en la edad moderna» (HAR2015-66579-P), financiado por el Ministerio de Economía y Competitividad.
} 


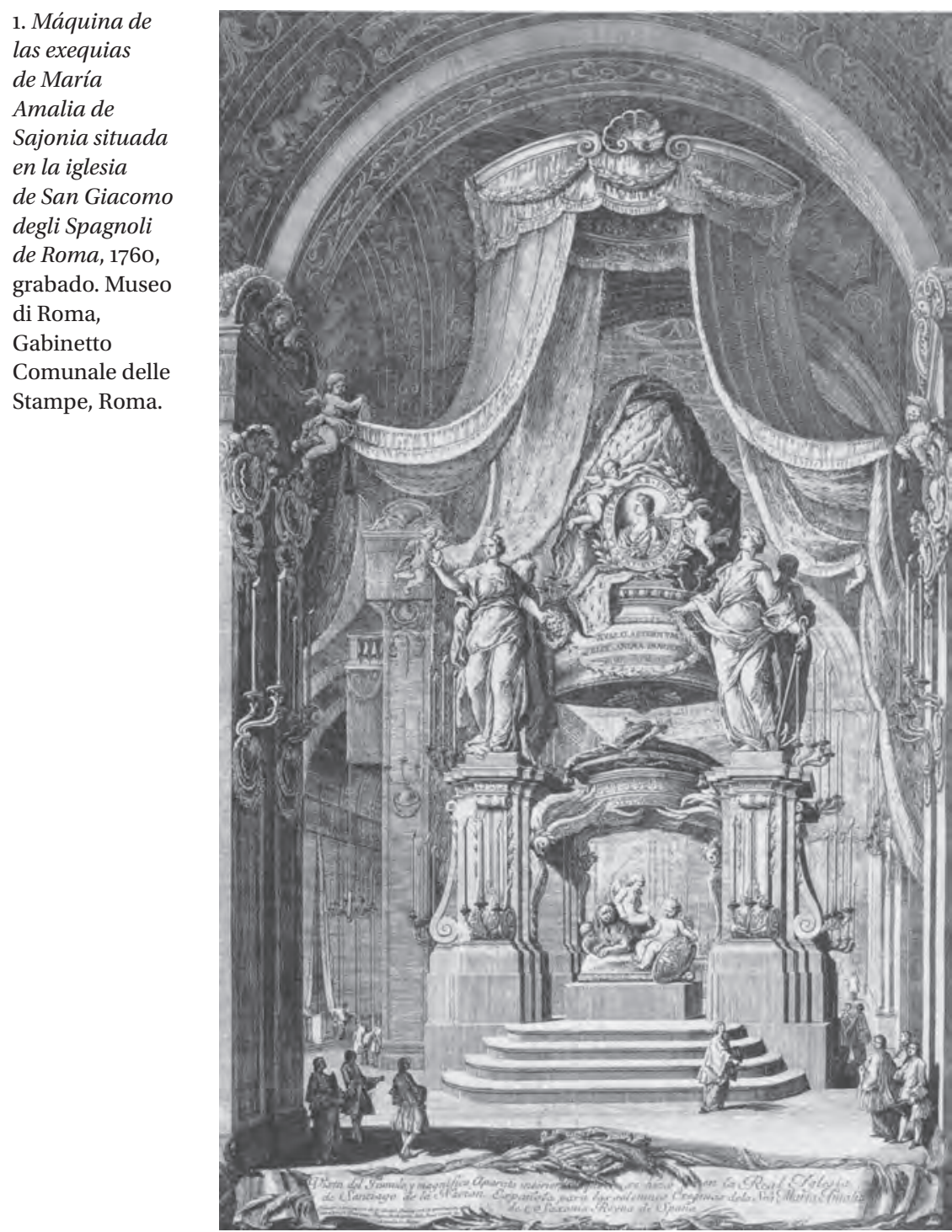

En el siglo XVIII, Roma era la meta más importante del Grand Tour, un destino prácticamente obligado para la mayoría de los artistas, eruditos y viajeros de toda Europa que esperaba culminar allí su periplo por el continente. En el caso de los artistas, la mayor parte de las veces este viaje tenía el sentido de ser la conclusión de sus procesos formativos y, de forma más bien excepcional, una posibilidad para desarrollar su carrera en Italia.

Una vez que los viajeros atravesaban la puerta de la plaza del Popolo, ya que la mayoría provenía del norte y viajaba hacia el sur, y tras inscribirse en la aduana que se encontraba en el templo de Adriano ubicado en la plaza di Pietra, comenzaba la búsqueda de alojamiento para quienes no lo hubiesen acordado antes de su llegada a Roma. Y a partir de entonces, los artistas extranjeros entraban a formar parte de esa gran escenografía en que se había convertido la ciudad y en la que cada nación se proyectaba como creía conveniente con el fin de deslumbrar al resto de los países presentes en ella, se convertían, de algún modo, en actores al servicio de sus países de origen (ilustración 1).

1. Caroli, F., Il Gran Teatro del Mondo. L'anima e il volto del Settecento, cat. exp., 13 de noviembre de 2003 - 12 de abril de 2014, Palazzo Reale, Milán. Milán: Skira, págs. 3-5. 
Aunque la mayoría de los especialistas que se han ocupado de los artistas extranjeros que pasaron un periodo en Roma ha tratado de buscar el lugar en que se alojaron, se advierte en la bibliografía la carencia de una visión de conjunto y de un análisis comparativo que permita establecer de qué manera la nacionalidad condicionó la elección de una parte u otra de la ciudad para vivir. Este tipo de análisis representa una cuestión extraordinariamente vasta, por lo que nos ceñiremos al periodo comprendido entre 1750 y 1780, quizá el momento álgido de la afluencia de extranjeros a Roma. Además, concentraremos nuestra atención en tres grupos, el de los franceses, los españoles y el conjunto formado por los artistas procedentes del norte de Europa, sobre el que será necesario realizar algunas matizaciones. ${ }^{2}$

\section{EL CASO FRANCÉS}

Muchos de los artistas franceses que llegaron a Roma entre 1750 y 1780 lo hicieron gracias a una pensión otorgada por la Académie Royale de Peinture et de Sculpture de París, que premiaba, de este modo, a sus alumnos más sobresalientes. Estos no necesitaron buscar alojamiento ya que gozaron del privilegio de residir en el palacio Mancini, sede de la Académie de France, sito en la via del Corso, que Francia alquiló en 1723, adquirió dos años más tarde y que mantuvo hasta $1793 .^{3}$ Es evidente que la finalidad del palacio Mancini no fue tan solo la de dar alojamiento a un limitado grupo de jóvenes artistas en un estadio inicial de sus carreras, sino que también fue un lugar de representación de este país. Frente a él desfilaba cada año la cabalgata de carnaval, en muchos casos organizada por los propios pensionados franceses, un espectáculo al que asistía toda la ciudad y que solo unos pocos privilegiados, elegidos según criterios de conveniencia política, podían ver desde el balcón del palacio. Además, buena prueba de la fuerte carga institucional del palacio Mancini es que todo el primer piso, denominado «apartamento del rey» y suntuosamente decorado con esculturas antiguas y tapices de Gobelins, era la zona en que se recibía a las grandes personalidades, mientras que el segundo piso estaba destinado al director y a sus pensionados, a los que se les dio las habitaciones peor iluminadas (ilustración 2).

Frente a la Académie de France se encontraba el palacio De Carolis, donde vivió, desde 1748 hasta su muerte en 1761, Claude François de Montboissier Beaufort-Canillac (1693-1761),

2. Lelo, K.; Travaglini, C.M., Roma nel Settecento. Immagini e realtà di una capitale attraverso la pianta di G.B. Nolli. Roma: CROMA, 2013; Rocciolo, D., «Gli stati delle anime di Roma», en Vodret, R., Alla ricerca di «Ghiongrat». Studi sui libri parrocchiali romanani (1600-1630). Roma: L'Erma di Bretschneider, 2011, págs. 9-16.

Este estudio se ha llevado a cabo a partir de un prolongado análisis de diversos tipos de documentación conservada en los archivos romanos. La más importante, dada la gran cantidad de información que aporta, son los estados de ánimas, registros de la comunión pascual que realizaba el párroco de cada una de las alrededor de ochenta parroquias romanas existentes en el periodo de tiempo que hemos establecido y que se conserva en el Archivio Storico del Vicariato, Roma (ASVR). Aunque se trata de una documentación muy irregular cuyo contenido depende del escrúpulo del párroco que la elaboraba, se suele indicar el nombre de los moradores de cada parroquia, su edad, nacionalidad, profesión y el estado civil. Desconocemos el tiempo que se tardaba en completar estos registros, aunque se puede suponer que iba desde los quince días hasta los dos meses, dependiendo de la magnitud de la parroquia. Tampoco sabemos si el párroco visitaba cada una de las casas para registrar a sus habitantes o si bien dejaban una especie de formulario que estos rellenaban para posteriormente dárselo al párroco correspondiente, incluso es posible que se alternasen estas dos modalidades de trabajo. La utilización de formularios es aludida en una carta que el marqués d'Aubeterre manda al conde de Saint-Florentin el 15 de junio de 1768 en la que se dice lo siguiente: «Il est vrai qu'on exige ici, de qui que ce soit, des billets pour la communion paschale, sous peine d'excommunication, au bout d'un certain tems, et d'être mis ensuite à l'Inquisition si on persiste» (DE MoNTAIGLON, A.; GuIFFreY, J., Correspondance des directeurs de l'Académie de France a Rome ave les surintendats des batiments. París: Noël Charavay, 1902, vol. XII, pág. 200, núm. 6033).

3. Guerci, M., Palazzo Mancini. Roma: Istituto Poligrafico e Zecca dello Stato, 2011. 


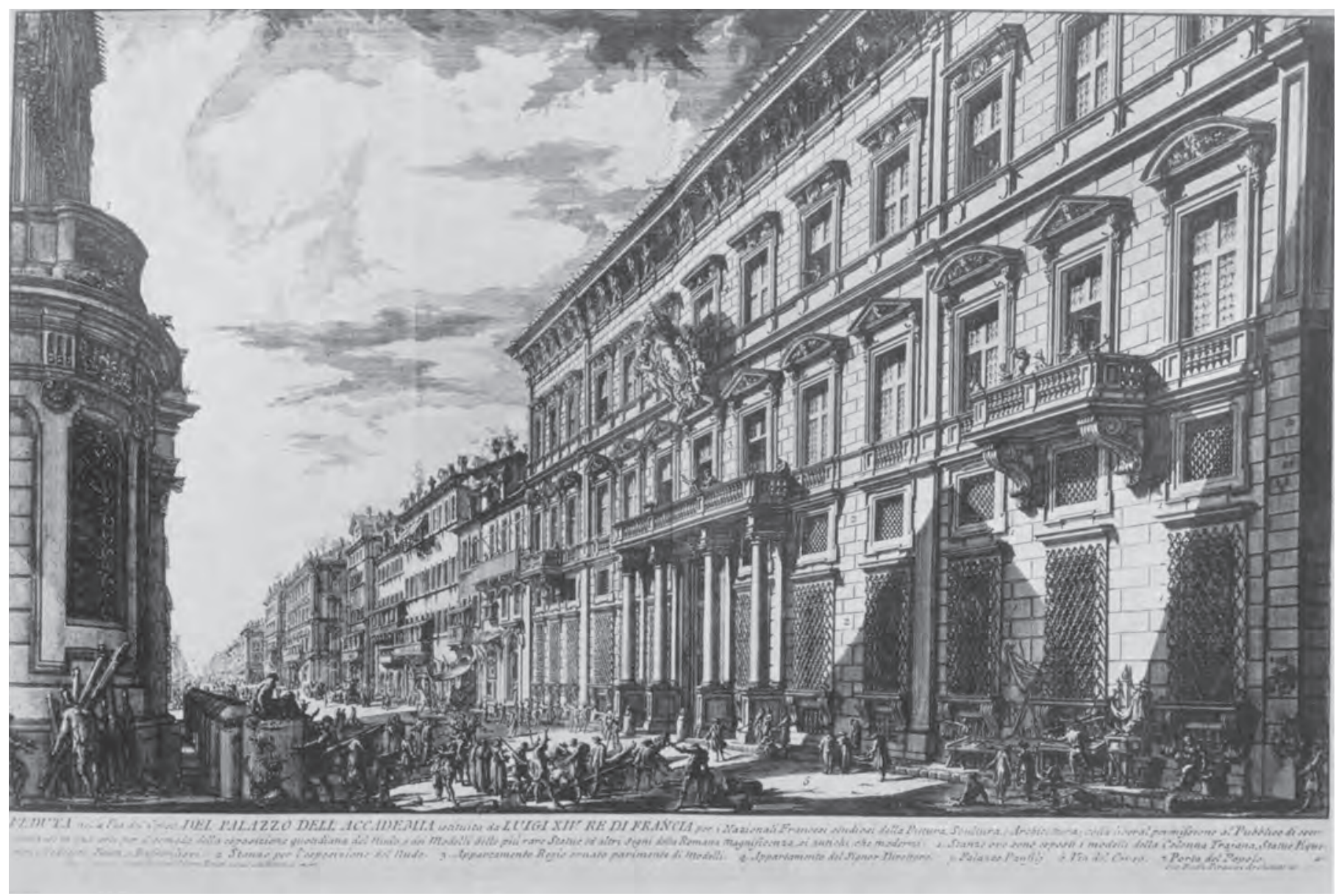

2. Giovanni Battista Piranesi Palacio Mancini, grabado para Vedute di Roma, 1752, aguafuerte, $40,6 \times 62 \mathrm{~cm}$. ministro interino del rey Luis XV y auditor de la Rota. ${ }^{4}$ El edificio pasó a convertirse en sede de la embajada de Francia en 1769, donde residió François-Joachim de Pierre, cardenal de Bernis (Saint-Marcel-d'Ardèche, 1715 - Roma, 1794), hasta $1794 .{ }^{5}$ Aunque el palacio De Carolis era ya un lugar esplendoroso gracias a la intervención de artistas de la talla de Sebastiano Conca (Gaeta, 1680 - Nápoles, 1764) y de Francesco Trevisani (Capodistria, 1656 - Roma, 1746), Bernis lo enriqueció aún más adecuándolo a su papel de espacio de representación de Francia en Roma.

Uno frente al otro, los palacios Mancini y De Carolis encarnaban la sólida posición de la nación francesa en el corazón de Roma, pero también en esa posición enfrentada de los edificios se sintetizaba la tensión existente entre ambos por acaparar un mayor protagonismo en la ciudad. De hecho, es posible que Bernis determinase establecer allí la embajada precisamente con la voluntad de imponerse a la Académie de France, cuyo protagonismo debía de parecerle excesivo. Ello podría explicar que el embajador encargase en 1771 un retrato suyo a uno de los pensionados de la Académie, Antoine-François Callet (1741-1823), que en 1772 se colgó

4. Giuggioli, A., Il palazzo de Carolis in Roma. Roma: Banco di Roma, 1980. El cardenal De Bernis destinó toda la primera planta del palacio De Carolis a los recibimientos, mientras que en la segunda se encontraban sus dependencias personales. El resto del edificio fue para su sobrina, la marquesa de Puy-Montbrun. Eran célebres los banquetes que tenían lugar entre los muros de este palacio y los jueves y los viernes se organizaban cenas, a las que asistían alrededor de mil personas, que podían terminar con un concierto. Uno de los personajes más insignes que pasó por el palacio De Carolis, tal y como señala Alfredo Giuggioli, fue Wolfgang Amadeus Mozart (Salzburgo, 1756 - Viena, 1791), quien habría tocado en él durante su paso por Roma en 1770 .

5. Montègre, G., La Rome des français au temps des Lumières. Roma: École française de Rome, 2011, págs. 199-220. 
en el palacio De Carolis, mientras que en 1773 se hallaba en la sala de los retratos del palacio Mancini. Además, la fuerte presencia del cardenal de Bernis en el palacio Mancini llegó hasta el punto de que intervino en la elección de algunos pensionados que tenían que ir a la Académie: en 1774, gracias a la recomendación de Bernis, un arquitecto elegido por él sustituyó al escultor Jean-Guillaume Moitte (París, 1746-1810), quien se vio obligado a abandonar el palacio Mancini por una enfermedad. ${ }^{6}$

Muy cerca del palacio Mancini y del De Carolis se halla el palacio Mellini, sito en la plaza de San Marcello, donde se estableció la librería de Bouchard y Gravier ${ }^{7}$ desde 1750 hasta 1795 - que también puede ser considerada un espacio representativo de la cultura francesa en Roma- ${ }^{8}$ En ella se vendían libros galos — posiblemente uno de sus mejores clientes fue el monasterio de Trinità dei Monti, donde existía una gran biblioteca- y guías de viaje adquiridas por los viajeros franceses del Grand Tour en la ciudad eterna, así como los grabados de Giovanni Battista Piranesi (Mogliano Veneto, 1720 - Roma, 1778). ${ }^{9}$ Además, debieron tener lugar en ella encuentros y tertulias de naturaleza cultural a las que no habrían faltado los pensionados de la Académie e incluso algunos viajeros franceses de paso por Roma (ilustración 3).

A la hora de confrontarnos con la cuestión del alojamiento de los artistas franceses en Roma, deberíamos interrogarnos sobre la función de sus iglesias nacionales, siendo la más importante de ellas San Luigi dei Francesi, fundada en 1478 con la vocación de tutelar a los peregrinos de lengua francesa y darles alojamiento cuando fuese oportuno en el hospital adyacente a la iglesia. Es necesario precisar que el antiguo principio de la natio gallicana, de naturaleza más lingüística que política, imponía que dicha iglesia-hospital no solo acogiese a los súbditos del rey de Francia, sino a todos los francófonos, lo que comprendía también el ducado de Lorena y Saboya. En los casos de San Nicola dei Lorenesi — una pequeña iglesia asentada en el espacio en que previamente se encontraba San Nicola en Agone, detrás de la plaza Navona-, y de Sant'Andrea y San Claudio dei Borgognoni, desde 1652 en la iglesia de San Claudio, próxima a la plaza de San Silvestro, junto a la que se creó un hospital, gozaron de una gestión autónoma hasta que en 1774 pasaron, junto a San Luigi, a estar bajo el control del cardenal de Bernis $\mathrm{y}$, por ende, de la corona francesa a la que aquel representaba en Roma.

La marcada vocación del palacio Mancini como espacio artístico debió mermar considerablemente el papel de lugares de acogida de los artistas francófonos de las iglesias-hospitales a las que nos hemos referido. De hecho, con respecto a otros países como España, los artistas franceses debieron recurrir en menos ocasiones a sus iglesias nacionales para establecerse en la ciudad eterna. Ello no limitó en absoluto su importancia en la celebración de las fiestas vinculadas a Francia, de las que se convirtieron en importantes escenarios.

En el caso de quienes no eran pensionados de la Académie o personajes afines a ella, de quienes llegaron a la ciudad eterna por su cuenta y riesgo, fueron realmente muy pocos los que recibieron el apoyo de la institución académica francesa y pudieron alojarse en el palacio Mancini. Uno de ellos fue Jean Robert Ango (? - Roma, 1773), un artista aún bastante desconocido,

6. Montègre, G., La Rome des français..., pág. 217; Archives du Ministére Français des Affaires Étrangères, Nantes (AMAE), Roma - Saint-Siège, carton 176, dossier VIII, carta de d'Angivillier a Bernis del 16 de septiembre de 1774.

7. Mancini, L., «La libreria Bouchard e Gravier di Roma. Profilo storico-documentario», La Bibliofilia, CXv, 2013, págs. 293-310.

8. Montègre, G., La Rome des français..., págs. 157-166, 171-181. Gilles Montègre señala la continua injerencia de la librería de Bouchard y Gravier en los asuntos de la Académie de France. Es más, durante los años ochenta la librería se encargó del transporte de obras de arte y de trabajos de los pensionados de la Académie desde Roma a Francia gracias a la colaboración de un personaje apellidado Luchetti, residente en Civitavecchia, desde donde los mandaba a la ciudad de Marsella.

9. Bonnard, F., Histoire du convent royal de la Trinité du Mont Pincio à Rome. París: A. Picard, 1933; BRULEY, Y. (dir.), La Trinité-des-Monts redécouverte. Art, foi et culture. Roma: De Luca, 2002. El monasterio de Trinità dei Monti, fundado en 1495 por Carlos VIII, comenzó a recibir visitas a lo largo de la segunda mitad del siglo XVIII de muchos viajeros que llegaron a Roma y deseaban contemplar El descendimiento de Daniele da Volterra y que, posiblemente también, se sintieron atraídos por el clima intelectual que se había creado en este lugar. 


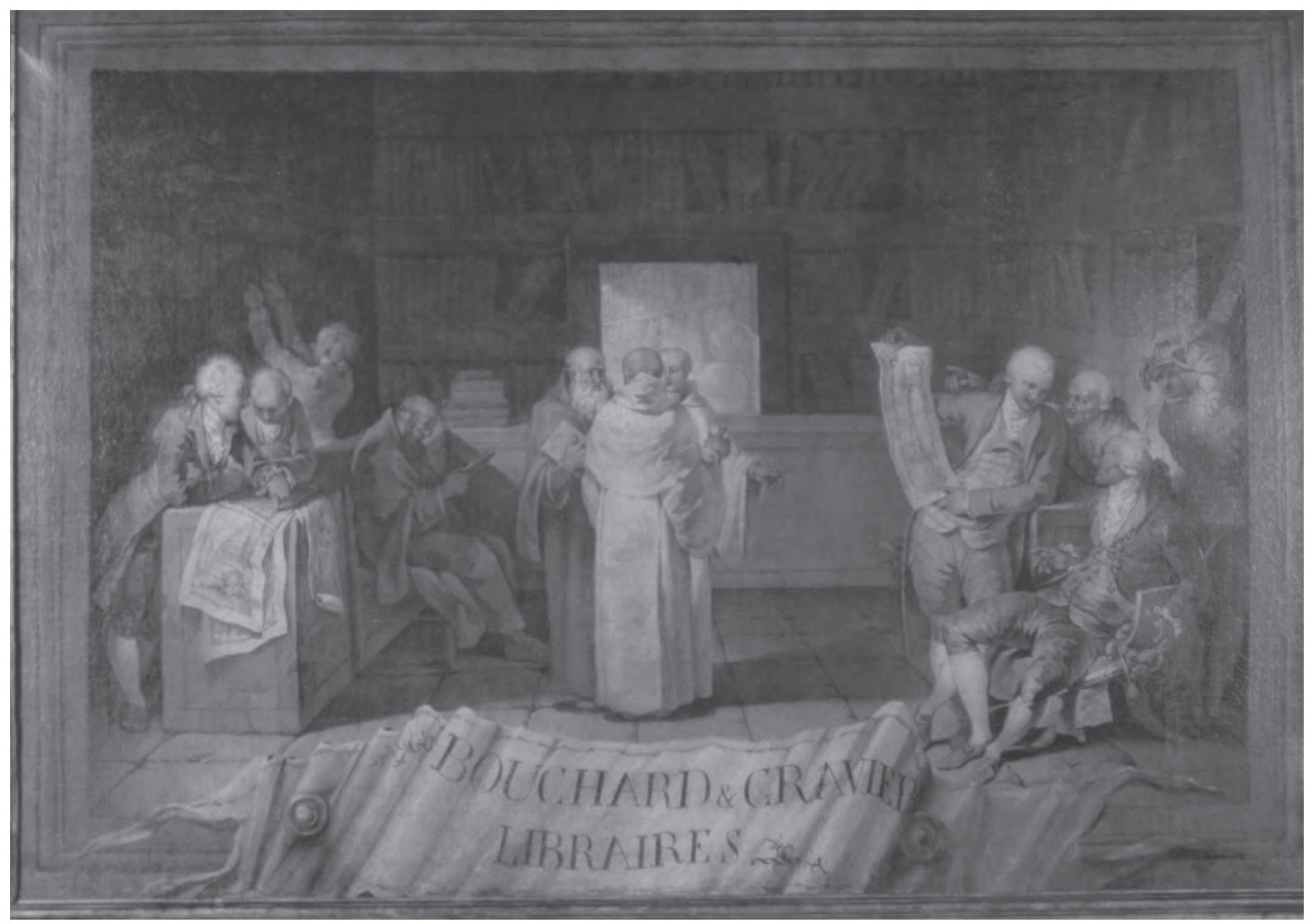

3. Anónimo Interior de la librería Bouchard et Gravier de Roma, c. 1770, óleo sobre tela, $125 \times 180 \mathrm{~cm}$. Colección privada. que estaba en Roma en torno a 1760 aunque probablemente habría llegado en $1750 .^{10}$ Aunque no sabemos si vivía en el palacio Mancini, podemos afirmar que en 1765 comía en él junto a otros pensionados de la Académie. El resto de los artistas franceses permanecieron en Roma tan solo cuando gozaron de un apoyo relevante, tal y como sucede con Julien de Parme (Cavigliano, 1736 - París, 1779), protegido de Guillaume du Tillot (Bayona, 1711 - París, 1774) o cuando, en calidad de artistas consagrados, consiguieron abrirse un hueco en el panorama artístico de la ciudad. En estos casos, eligieron los domicilios que se encontraban en las parroquias más céntricas de Roma, especialmente en Sant'Andrea delle Fratte.

Asimismo, el hecho de que un artista francés no se encontrase vinculado a la Académie, y no estuviese, por tanto, obligado a rendir cuentas de su formación en Roma, debió de convertir a muchos de ellos, tal y como señala Montegre, en extraordinarios cicerones de la ciudad

10. Ananof, A., «Fragonard et Ango, collaborateurs de Saint-Non», Bulletin de la Société de l'Histoire de l'Art Français, 1962, págs. 117-120; BoYER, S., «Quelques propositions autour de Jean-Robert Ango (? - après le 16 janvier 1773)», Les cahiers d'histoire de l'art, 6, 2008, págs. 88-103. Debieron de ser las grandes dotes de dibujante de Ango, además de su nacionalidad y la intercesión del abad Saint-Non (1759-1761), lo que propició que acompañase a Jean-Honoré Fragonard a Nápoles. En cualquier caso, cabría señalar que la implicación de la Académie en la estancia de Ango en Roma debió ser relativa ya que, como informa el pintor Julien de Parme en una carta que escribe a un pintor belga que conoció en el palacio Farnese, Ango sufrió en 1772 una apoplejía que le impidió practicar la pintura hasta tal punto que, en 1773, mendigaba por las calles de la ciudad. Este dato nos hace pensar que la Académie ayudó o dio cobijo a aquellos personajes que tenían cierta importancia o que eran recomendados por figuras de relevancia. 
que orientaron a viajeros franceses. Estos últimos se alojaron en pensiones regentadas por compatriotas como la que abrió Charles Roland frente a la embajada española, en la plaza de Spagna, a la que llamó Ville de Londres. ${ }^{11}$ De hecho, algunos franceses que viajaron a la ciudad eterna recurrieron a ellas, al menos en un primer momento, tal y como hizo François-de-Paule Latapie (Burdeos, 1739-1823), quien llegó a Roma el 24 de marzo de 1775 sin un lugar donde quedarse. Por mediación del cochero que le llevó hasta la ciudad eterna, también francés, entró en contacto con una locanda ubicada en la via della Croce, en las proximidades del palacio de Spagna, regentada por un personaje oriundo de Lorena que le proporcionó una habitación y la cena a buen precio. Esta no era la única locanda francesa que había en la via della Croce, ya que también allí se encontraba una especie de albergue en el que residió durante un tiempo Jean-Marie Roland de la Plattère (Thizy, Ródano-Alpes, 1734 - Bourg-Beaudoin, Normandía, 1793) durante su permanencia en la ciudad eterna en 1777 .

\section{LOS ESPAÑOLES EN ROMA}

En comparación con el caso francés, resulta más complejo explicar las modalidades de alojamiento de los artistas españoles, en su mayoría jóvenes en periodo de formación, posiblemente porque las circunstancias que rodearon a la concesión de las pensiones para ir Roma eran ostensiblemente más complejas que las que concernían a los artistas galos. De hecho, hasta la creación de la Real Academia de España en 1873, y pese a los infructuosos intentos del tutor de los pensionados, Francisco Preciado de la Vega (Sevilla, 1712 - Roma, 1789), de que los jóvenes españoles se alojasen todos juntos en la iglesia-hospital de San Giacomo degli Spagnoli, ${ }^{12}$ no existió un espacio análogo al palacio Mancini que sirviese para acoger a quienes iban a Roma.

La búsqueda de los artistas españoles en los stati delle anime (estados de ánimas) resulta infructuosa en buena parte de los casos durante los primeros años de permanencia en Roma. Ello nos permite suponer que hubiesen podido recurrir a las iglesias-hospitales nacionales españolas, San Giacomo degli Spagnoli, en la parroquia de Sant'Eustachio, y Santa Maria di Monserrato degli Spagnoli, en la de Santa Caterina della Rota, para residir un breve tiempo, quizá menos de un año. Buen ejemplo de ello es Domingo Candau, quien solicitó una pensión extraordinaria a la Real Academia madrileña, tal y como se refleja en la junta ordinaria del 9 de septiembre de 1773, documentación en la que también se precisa que transcurrió buena parte

11. En los años cincuenta del siglo XVIII se constata la presencia en Roma de alrededor de doscientos treinta albergues u hoteles de los que la mayoría se encontraban en los rioni de Campo Marzo, Ponte, Colonna y Borgo. En realidad, la concentración más significativa de albergues se dividía entre la plaza del Popolo y la plaza de Spagna, puesto que era la parte de Roma que con mayor frecuencia transitaron los viajeros que llegaban a la ciudad eterna.

12. Archivo de la Real Academia de Bellas Artes de San Fernando, Madrid (ARABASF), Juntas ordinarias, generales y públicas, 3/82, Junta ordinaria, 27 de julio de 1762. Ante la petición de Preciado de la Vega la Real Academia de Bellas Artes de San Fernando responde de manera evasiva. Muchos años después, cuando Preciado de la Vega escribió su Arcadia pictórica, el sevillano vuelve a reivindicar la necesidad de crear un espacio común en el que poder controlar adecuadamente la formación de los jóvenes españoles (PRECIADO DE LA VEGA, F., Arcadia pictórica en sueño: alegoría ó poema prosaico sobre la teoría y la práctica de la pintura. Madrid: Antonio Sancha, 1789, págs. 199-200): «Con todo yo desearía, que se perfeccionasen tales disposiciones con establecer una casa ó Colegio, en que unidos, y separados con unas prudentes leyes viviesen juntos los Pensionados, gobernados por un prudente y habil director, que velase sobre su aplicación y costumbres, ya que facilmente los jovenes con la libertad, que separados logran, viciarse y descuidar en la carrera de la profesión, á que se dedican. Asi lo estableció el gran Luis decimo quarto Rey de Francia por medio de su ministro Colbert en Francia». 
de su estancia en Roma en la iglesia-hospital de Santa Maria di Monserrato. ${ }^{13}$ Incluso es posible suponer que el escultor Juan Adán (Tarazona, 1741 - Madrid, 1816) -algo que es válido también para su compañero Francisco de Goya (Zaragoza, 1746 - Burdeos, 1828) durante los dos años que este permaneció en Roma (1769-1771) - hubiese vivido en la iglesia-hospital aragonesa. Ello podría explicar la comisión de la escultura Santa Isabel de Portugal que el turiasonense concluyó en 1772, prueba de su relación con la fundación aragonesa. ${ }^{14}$

Una vez resuelta la llegada a Roma, muy probablemente gracias al apoyo de estas instituciones hospitalarias o de algunas estructuras conventuales, la mayor parte de los artistas españoles se irán aproximando a la zona de la plaza Barberini, en la parroquia de Santa Susanna. Allí residió prácticamente durante toda su vida Preciado de la Vega, a excepción de los primeros años en Roma durante los que se asentó en la parroquia de Sant'Andrea delle Fratte en compañía de Felipe de Castro. De este modo, se podría decir que la parroquia de Santa Susanna, en la que no se constata una significativa densidad de artistas, se convirtió en uno de los espacios más españoles de la ciudad durante los años en que Preciado vivió allí. Estos se alojaron en casas que se encontraban muy cerca de la del sevillano, aunque en algunos casos también residieron, posiblemente por mediación de Preciado de la Vega, en conventos que se encontraban muy cerca de la plaza Barberini ${ }^{15}$ (ilustración 4 ).

Vivir en la parroquia de Santa Susanna debió de ser una circunstancia particularmente importante para los escultores españoles en periodo de formación e incluso para su posterior inserción en el mercado artístico romano. Ello podría deberse a la presencia en ella de los talleres de dos de los escultores más sobresalientes del panorama artístico, Filippo della Valle (Florencia, 1698 - Roma, 1768) y Agostino Penna (Roma, 1735-1811). La ubicación de ambos en esta parroquia no deja de ser un tanto peculiar, ya que la mayoría de los escultores eligieron espacios que se encontraban en las inmediaciones del puerto de Ripetta, adonde llegaba el mármol. Buena prueba de la intensidad de los vínculos de algunos artistas españoles con Della Valle es que Juan Adán y Gabriel Durán se casaron en 1776 con dos de sus hijas, Violante y Petronilla, tal y como atestigua la documentación de la parroquia de Santa Susanna. ${ }^{16}$

Durante los últimos años de su pensión, quizá gracias a la obtención de comisiones artísticas o a la consolidación de amistades de cierta relevancia, fruto de una dilatada permanencia en Roma, varios artistas españoles abandonaron la parroquia de Santa Susanna para trasladarse a la de Sant'Andrea delle Fratte, en la que se aprecia una elevadísima concentración de pintores y escultores, algunos de ellos muy importantes en el panorama romano. En esta parroquia se produjo la convivencia de artistas procedentes de diferentes naciones y en ella se sitúa el palacio de Spagna, sede de la embajada, que debió de constituir una referencia fundamental para los españoles en Roma. Llegados a este punto, es necesario recordar que entre el

13. ARABSF, Juntas particulares, ordinarias, generales y públicas, 1770-1775, sig. 3/83, junta ordinaria, 9 de septiembre de 1773 .

14. Gallego, R., «Sobre las capitulaciones matrimoniales de Goya y la prisa por abandonar Roma», Archivo Español de Arte, 87, 346, 2014, págs. 109-118.

15. Preciado de la Vega mantuvo una importante amistad con Giovan Battista Ponfreni (Roma, 1715-1795), director de la fábrica pontificia de mosaicos, tal y como se advierte en una carta enviada por el sevillano en 1765. De esta se desprende que Ponfreni y Preciado se estimaban y que podrían haberse conocido en Roma durante su juventud llegando a mantener una intensa relación, ya que ambos vivieron en la plaza Barberini. Mientras que Preciado compartía su casa con su esposa Caterina Cherubini, Ponfreni vivía en el convento de los capuchinos, que se encontraba en la hoy denominada via Veneto y del que tan solo se conserva la iglesia, donde deseaba ser enterrado, tal y como se puede verificar en su testamento del 28 de enero de 1792 en el que hacen las veces de testigos siete frailes del convento, abierto el 21 de enero de 1795 ante Bernardino Nocchi (ASR, 30 notai capitolini, ufficio 12, Megliorucci). Incluso se podría sospechar que este convento hubiese sido en algún momento una solución de alojamiento para algunos artistas que estaban bajo la tutela de Preciado gracias a la intercesión de Ponfreni. De hecho, cuando el pintor Domingo Álvarez Enciso tuvo problemas con la Real Academia de Bellas Artes de San Fernando debido a su conducta, se le destinó al convento de San Basilio, en la parroquia de Santa Susanna, donde residió al menos durante el año 1761.

16. ASVR, Santa Susanna, Licenze matrimoniali, 4, 1761-1790; ASVR, Santa Susanna, Liber matrimoniorum, 3, 1774-1810.

$48-$ ARticles 


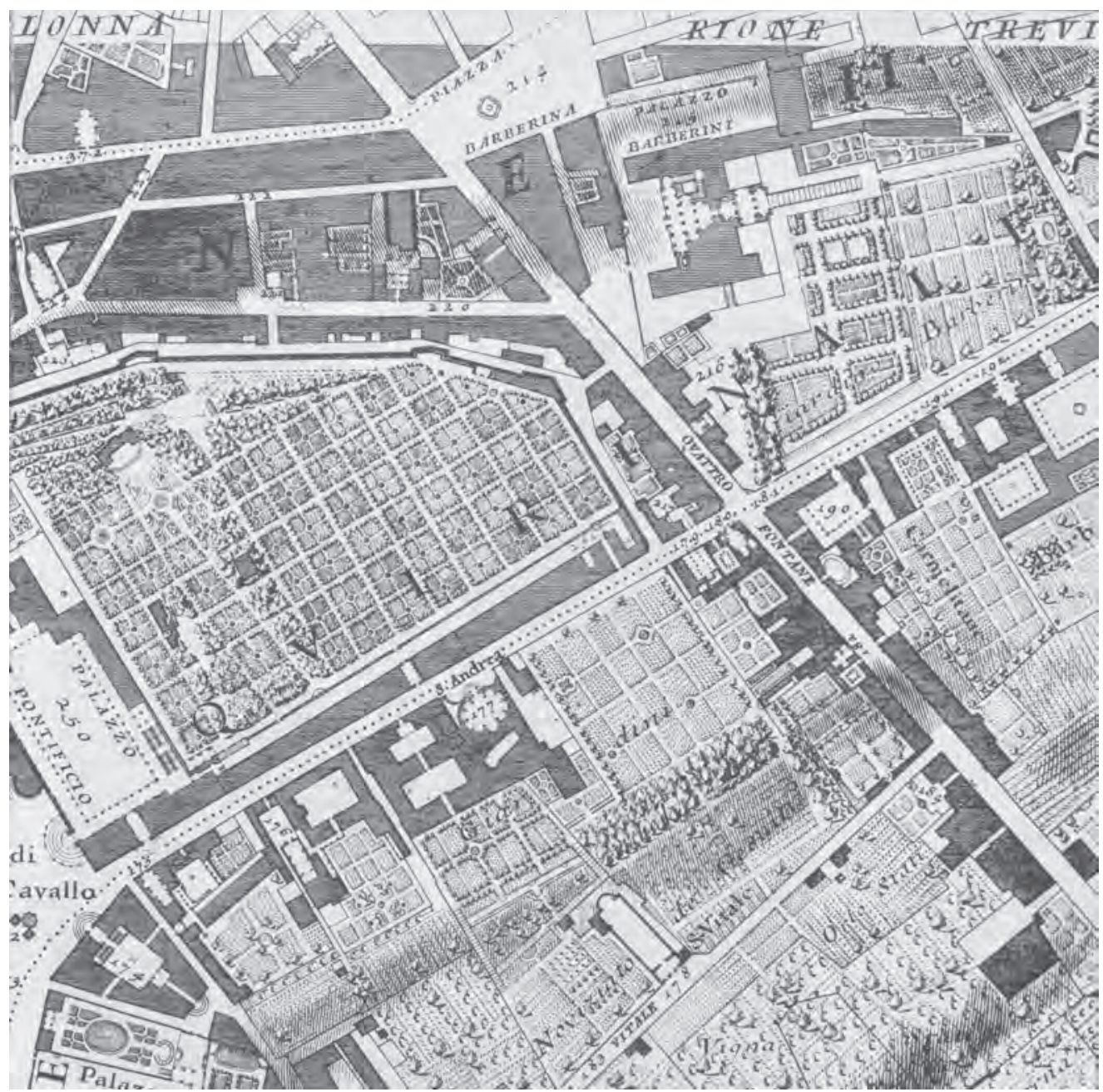

4. Giovanni Battista Nolli Nueva planta de Roma (detalle), 1748, grabada por Giuseppe Vasi, Rocco Pozzi, Pietro Campana y Carlo Nolli según dibujos de Giovanni Battista Nolli, Giovanni Paolo Pannini y Stefano Pozzi, aguafuerte y buril sobre papel. Istituto Nazionale per la Grafica, Roma.

palacio de Spagna y la iglesia de Trinità dei Monti, mandada construir por el monarca francés Carlos VIII para la orden de los mínimos de San Francisco de Paula, se encuentra la escalinata (1721-1723) que Francia encargó a Alessandro Specchi (Roma, 1668-1729). Se trata de una obra que conmemora la paz entre este país y España aunque, contemporáneamente, encarna las tensiones que aún existían entre ambas potencias y en torno a la cual, tanto Francia como España, celebraron sus fiestas con fastuosas máquinas (ilustración 5).

\section{Artistas Provenientes DEL NORTE DE EUROPA}

Los artistas provenientes del norte de Europa, sin duda alguna la presencia más numerosa de entre los extranjeros que visitaron Roma, se podrían dividir en dos categorías que en raras ocasiones se llegarán a mezclar. ${ }^{17}$ La primera de ellas estaría integrada por quienes conduje-

17. Para el estudio de los artistas británicos, además del estudio de los estados de ánimas, se ha consultado el texto de Ingamells, J., A Dictionary of British and Irish Travellers in Italy: 1700-180o. New Heaven - Londres: Yale University Press, 
5. Giovanni

Battista Nolli

Nueva planta

de Roma

(detalle), 1748,

grabada por

Giuseppe Vasi,

Rocco Pozzi,

Pietro Campana

y Carlo Nolli

según dibujos

de Giovanni

Battista Nolli,

Giovanni Paolo

Pannini y Stefano

Pozzi, aguafuerte

y buril sobre

papel. Istituto

Nazionale

per la Grafica,

Roma.

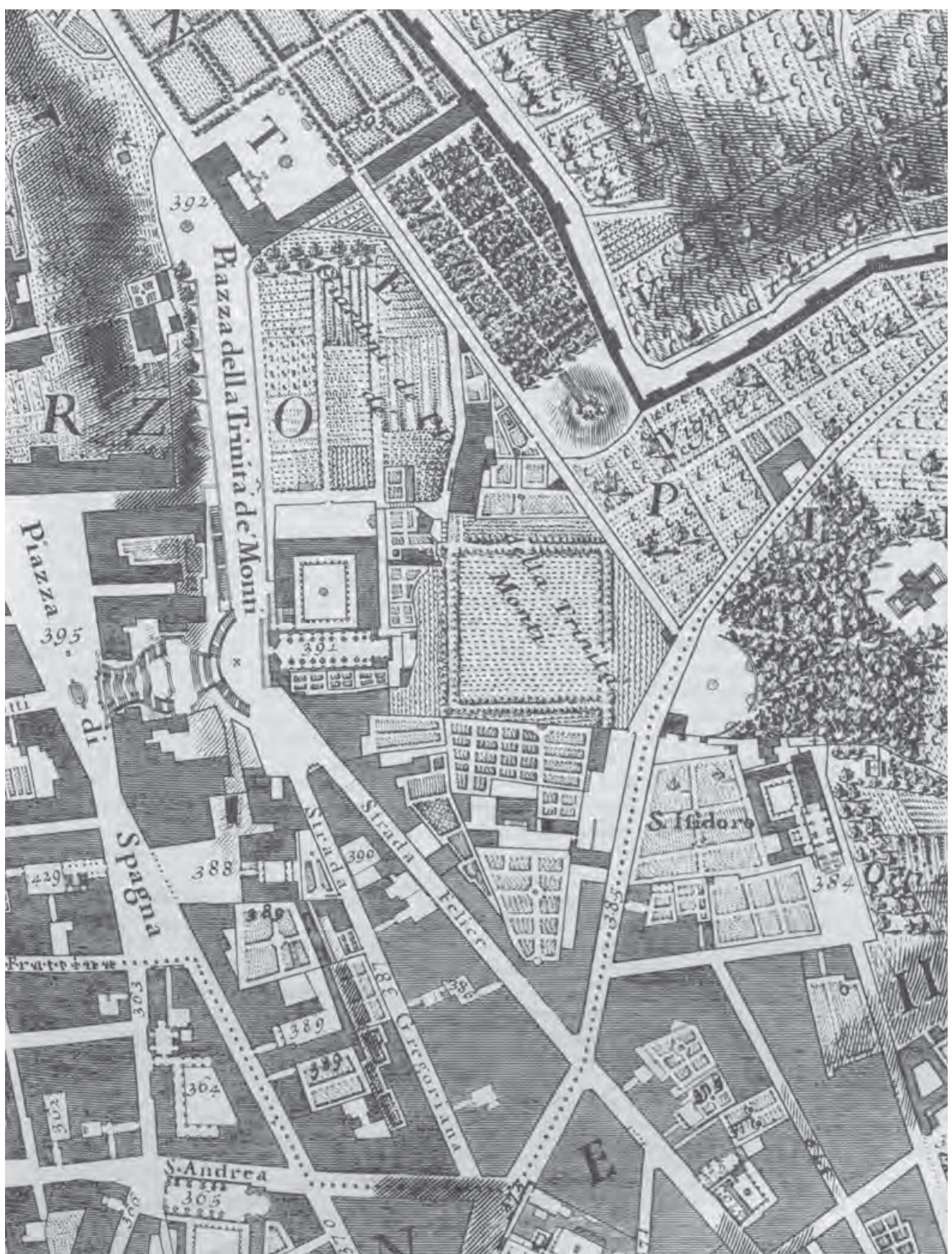

ron su estancia en Roma de manera libre e independiente, de espaldas a las instituciones oficiales, como un grupo bastante homogéneo aunque un tanto hermético que no manifestó interés por relacionarse con artistas que estaban adscritos a la órbita académica ni por entrar en el mercado artístico de la ciudad. El mejor representante de esta categoría de artistas provenientes del norte de Europa podría ser el suizo Johann Heinrich Füssli (Zúrich, 1741 - Putney Hill, Londres, 1825), en Roma entre 1770 y 1777, quien atrajo, entre otros, al escocés John Brown (Edimburgo, 1752 - Leith, 1787), en Italia desde 1769, al danés Nikolai Abraham Abildgaard

1997. Se trata de un extraordinario compendio de los artistas de esta nacionalidad en el que se sigue su trayectoria por Italia a partir del análisis de una amplia variedad de fuentes de información. 
(Copenhague, 1743 - Frederiksdal, 1809), estable en la ciudad eterna entre 1772 y 1777, el sueco Johan Tobias Sergel (Estocolmo, 1740-1814), y el escocés Alexander Runciman (Edimburgo, 1736-1785), quien fue a Italia en 1769 y allí permaneció hasta 1772. Casi todos ellos, aunque de manera especial Füssli, se caracterizaron por su profunda formación literaria así como por su aproximación a la pintura manierista y por el redescubrimiento de Miguel Ángel. Además, se confrontaron con la escultura clásica no desde un punto de vista puramente formal o con una finalidad comercial o académica, sino que la analizaron en cuanto vehículo para la expresión de las inquietudes y las pasiones del ser humano. La mayoría de ellos no parecían interesados en adquirir obras de arte, quizá también porque no tenían unas condiciones económicas que se lo permitiesen.

Por otra parte, en Roma se dieron cita artistas, en su mayoría británicos, muchos de ellos de buena familia o muy bien relacionados, que se introdujeron con cierta facilidad en la élite cultural y artística romana, que participaron activamente en el mercado anticuario de la ciudad y que echaron raíces en Roma, ya que muchos de ellos permanecieron allí hasta el final de sus días. ${ }^{18}$ Dentro de este grupo podríamos colocar, entre otros muchos, a Gavin Hamilton (Lanarkshire, 1723 - Roma, 1798), que llegó a Roma en torno a 1740, al escocés David Allan (Alloa, 1744-1796), en Roma entre 1764 y 1777, y a Joshua Reynolds (Plympton St. Maurice, 1723 - Londres, 1792), en Italia entre 1749 y 1752. Muchos de ellos adquirieron obras de arte, generalmente clásicas, que se llevaron a sus países de origen y que fueron de extraordinaria utilidad en el nacimiento y en el desarrollo de las academias de Bellas Artes; con esta aportación de yesos y piezas grecorromanas suplieron la enorme laguna de arte clásico que existía en tierras británicas. Además, muchos de ellos se hicieron retratar por Pompeo Batoni (Lucca, 1708 - Roma, 1787), especializado en este tipo de caros souvenirs del Grand Tour.

Los artistas que forman parte del primer grupo se establecieron, en buena parte de los casos, en la via del Babuino o muy cerca de ella, eligiendo el área de esta calle que se divide entre la parroquia de Santa Maria del Popolo y la de Sant'Andrea delle Fratte. Uno de los primeros en hacerlo fue Füssli, asentado allí entre 1770 y 1776 y, probablemente, debieron ser las relaciones de amistad que fue entablando el suizo lo que motivó que muchos de los artistas que formaban parte de su órbita pasasen por esta parte de la ciudad. La mayoría de ellos realizaron el viaje a Roma por su cuenta y riesgo, sin apoyo económico alguno, por lo que las relaciones de amistad y la solidaridad de artistas pertenecientes a un mismo círculo y con análogos intereses debieron de tener una importancia fundamental (ilustración 6).

En el caso de aquellos artistas que formaron parte activa del mundo artístico, pero sobre todo del mercado anticuario de la ciudad eterna, se aprecia una clara tendencia a establecerse en la via Felice, hoy via Sistina, que desembocaba en la plaza Barberini, con una preferencia por los palacios Tomati, Nazzari y Zuccari, dentro de la parroquia de Sant'Andrea delle Fratte. La razón podría ser su pertenencia a la alta sociedad, lo que significaba, esencialmente, una situación económica desahogada que les permitía pagar el alquiler de algunos de los edificios más importantes de Roma donde, asimismo, era más fácil entablar relaciones con los artistas más relevantes de la ciudad, lo que debió de incidir en la manera en que se desarrolló su estancia en Italia. Además, no deberíamos olvidar que en el palacio Tomati vivió Piranesi durante la mayor parte de su vida en Roma, un personaje clave en el mercado anticuario romano que habría enlazado a los ingleses con este mundo propiciando la adquisición de obras, especialmente de arte clásico, y no muy lejos de allí se encontraba el taller de Bartolomeo Cavacep-

18. Archivio di Stato di Roma, Roma (ASR), 30 notai capitolini, Sacchi, 17 de febrero de 1797. Una prueba del arraigo en Roma de una parte de los viajeros del norte de Europa la encontramos en el caso de Thomas Harwood (c. 1774 - Roma, 1798), de procedencia inglesa, quien, según se deduce de la documentación archivística, poseía una tienda en la via della Croce en la que vendía terraglie d'Inghilterra, es decir, cerámica inglesa. 
6. Giovanni

Battista Nolli

Nueva planta

de Roma

(detalle), 1748,

grabada por

Giuseppe Vasi,

Rocco Pozzi,

Pietro Campana

y Carlo Nolli

según dibujos

de Giovanni

Battista Nolli,

Giovanni Paolo

Pannini y Stefano

Pozzi, aguafuerte

y buril sobre

papel. Istituto

Nazionale

per la Grafica,

Roma.

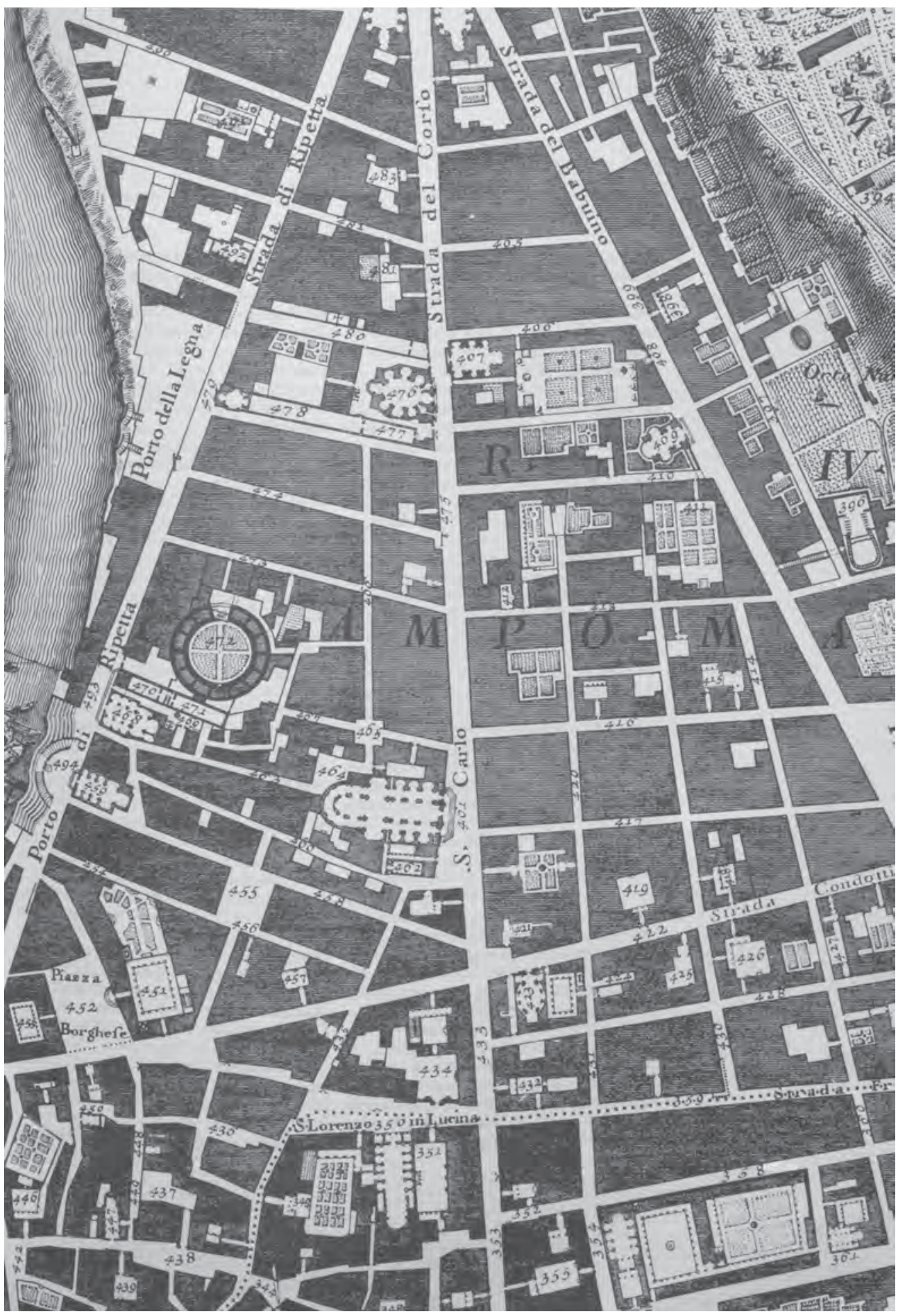

pi (Roma, 1716-1799), restaurador de obras clásicas y fuente vital para la adquisición de este tipo de piezas.

El Caffè degli Inglesi, en la plaza de Spagna, era por su estratégica posición un lugar en el que no solo se dieron cita los artistas de esta nacionalidad, sino que también pasaron por él artistas de otros países. Este espacio decorado por Piranesi, que ideó para él imágenes inspiradas en el antiguo Egipto, fue transitado de manera habitual por Francesco Ravai, más conocido 
como el «nano Bajocco». El hecho de que fuese retratado por artistas de las más diversas procedencias, como por ejemplo Jean-Honoré Fragonard (Grasse, 1732 - París, 1806), Jean Grandjean (Ámsterdam, 1752 - Roma, 1781) y Nikolai Abraham Abildgaard, demuestra que el Caffè degli Inglesi fue uno de los espacios, además de las academias públicas y privadas, en que se habrían podido entablar relaciones trascendentales para el desarrollo de la estancia de los artistas en Roma o para su proceso de maduración artística. ${ }^{19}$

19. Jannattoni, L., «... Il “Caffè Nuovo” lo "Scalinone” e il nano Bajocco», en Pietrangeli, C.; Liverani, P., Palazzo Ruspoli. Roma: Editalia, 1992, págs. 351-382; VAlverde, I., «Sublime heterodoxia: Henry Fusli y su círculo en Roma», en SuREDA, J. (coord.), Goya e Italia, cat. exp., 1 de junio - 15 de septiembre de 2008, Museo de Zaragoza, Zaragoza. Madrid: Turner - Fundación Goya en Aragón, 2008, págs. 157-169. Además de Francesco Ravai, existió después de él otro enano que también recibió la denominación de Bajocco, Giovanni Giganti. A diferencia del primero, el segundo se caracterizaba por sus vestiduras cuidadas mientras que Ravai, tal y como se puede advertir en los muchos retratos que de él se conservan, iba siempre vestido con andrajos. 